INPLASY

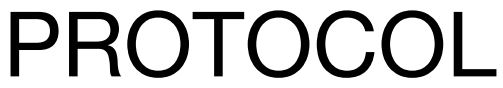

To cite: Gupta et al.

Monotherapy with platelet-rich plasma for male and female pattern baldness: protocol for a systematic review. Inplasy protocol 202170088. doi: 10.37766/inplasy2021.7.0088

Received: 27 July 2021

Published: 27 July 2021

Corresponding author: Aditya Gupta

agupta@mediproberesearch.com

Author Affiliation:

Mediprobe Research Inc.

Support: Self-financed.

Review Stage at time of this submission: Preliminary searches.

Conflicts of interest: None declared.

\section{Monotherapy with platelet-rich plasma for male and female pattern baldness: protocol for a systematic review}

Gupta, AK¹; Talukder, M²; Bamimore, MA³.

Review question / Objective: To gather evidence on the effect of monotherapy with platelet-rich plasma for androgenetic alopecia in men and women.

Condition being studied: Pattern baldness, also referred to as androgenetic alopecia.

Eligibility criteria: Randomized and observational studies that investigated the impact of monotherapy with platelet-rich plasma on male and female pattern baldness. Only evidence in English will be included; there will be no date restrictions.

INPLASY registration number: This protocol was registered with the International Platform of Registered Systematic Review and Meta-Analysis Protocols (INPLASY) on 27 July 2021 and was last updated on 27 July 2021 (registration number INPLASY202170088).

\section{INTRODUCTION}

Review question / Objective: To gather evidence on the effect of monotherapy with platelet-rich plasma for androgenetic alopecia in men and women.

Rationale: Globally, millions are diagnosed with androgenetic alopecia-which is also known as 'pattern baldness'. Though clinically benign, persons with this condition seek therapy as hair loss is associated with negative psychosocial consequences. Topical minoxidil (5 and 2 per cent) and oral finasteride $(1 \mathrm{mg} /$ day) are long-standing drugs for pattern baldness; however, platelet-rich plasma is a more recent therapy. Evidence synthesis of the platelet-rich plasma literatureinsofar as male and female pattern baldness-would be useful to clinicians, patients and researchers.

Condition being studied: Pattern baldness, also referred to as androgenetic alopecia. 


\section{METHODS}

Search strategy: PubMed 262 (("baldness"[Title/Abstract] OR "hair loss"[Title/Abstract] OR "alopecia"[Title/ Abstract]) OR (alopecia[MeSH Terms])) AND (("platelet"[Title/Abstract] AND "rich"[Title/Abstract] AND "plasma"[Title/ Abstract]) OR (platelet rich plasma[MeSH Terms])); Web of Science 290 (TS=("platelet" AND "rich" AND "plasma")) AND TS=("hair loss" OR "alopecia" OR bald*); EMBASE (Ovid) 389 Embase Classic+Embase 1 ("hair loss" or "alopecia" or bald*).ab.; 2 exp alopecia/ ; 3 1 or 2; 4 exp thrombocyte rich plasma/ ; 5 ("platelet" and "rich" and "plasma").ab. ; 6 4 or 5 ; 73 and 6 ; Scopus 204 ( ABS ("hair loss" OR "alopecia" OR bald* ) AND ABS ( "platelet" AND "rich" AND "plasma" ) ); CINAHL 65 \# Query Results S7 S4 AND S6 65; S6 S1 OR S5 2,740; S5 (MH "PlateletRich Plasma+") 1,834; S4 S2 OR S3 4,775; S3 AB "alopecia" OR "hair loss" OR bald* 2,948 ; S2 (MH "Alopecia+") 2,989; S1 AB "platelet" AND "rich" AND "plasma" 1,978.

Participant or population: Subjects diagnosed with androgenetic alopecia; participants of all ages and races/ ethnicities are included.

Intervention: Monotherapy with Plateletrich plasma.

Comparator: Comparators can include: (1) no treatment (i.e., nothing), (2) other monotherapies, and (3) placebo.

Study designs to be included: Evidence will be gathered from randomized and observational studies.

Eligibility criteria: Randomized and observational studies that investigated the impact of monotherapy with platelet-rich plasma on male and female pattern baldness. Only evidence in English will be included; there will be no date restrictions.

Information sources: Searches were conducted in CINAHL, EMBASE (Ovid), PubMed, Scopus, and Web of Science.
Main outcome(s): The outcome(s) will be consolidated according to the evidence we gather.

Additional outcome(s): None.

Data management: Data will be organized into spreadsheets.

Quality assessment / Risk of bias analysis: Quality of evidence within studies will be assessed using Cochrane Collaboration's risk of bias (RoB) tool; evidence quality across studies will be evaluated using the Grading of Recommendations, Assessment, Development and Evaluations (GRADE) framework.

Strategy of data synthesis: The evidence we gather will guide all quantitative analyses; if there is sufficient data, metaanalyses will be conducted.

Subgroup analysis: If there is sufficient data, clinically meaningful subgroup analyses will be done.

Sensitivity analysis: None.

Language: Only evidence in English language will be included.

Country(ies) involved: Canada.

Keywords: platelet-rich plasma; pattern baldness; hair loss; alopecia.

Contributions of each author:

Author 1 - Aditya Gupta.

Email: agupta@mediproberesearch.com

Author 2 - Mesbah Talukder.

Email: mtalukder@mediproberesearch.com

Author 3 - Mary Bamimore.

Email: mbamimor@alumni.uwo.ca 